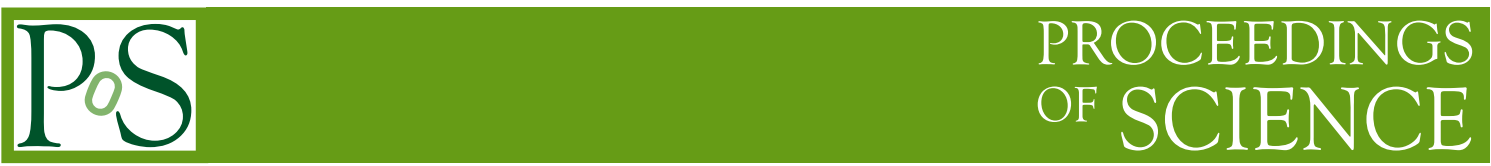

\title{
Constraining Sterile Neutrinos from Precision Higgs Data
}

\section{Arindam Das}

School of Physics, KIAS, Seoul 02455, Korea E-mail: arindamekias.re.kr

\begin{abstract}
We use the LHC Higgs data to derive updated constraints on electroweak-scale sterile neutrinos that naturally occur in many low-scale seesaw extensions of the Standard Model to explain the neutrino masses. We also analyze the signal sensitivity for a new final state involving a single charged lepton and two jets with missing energy, which arises from the decay of sterile neutrinos produced through the Higgs and $W, Z$ boson mediated processes at the LHC. Future prospects of these sterile neutrino signals in precision Higgs measurements, as well as at a future $100 \mathrm{TeV}$ collider, are also discussed.
\end{abstract}

39th International Conference on High Energy Physics

4-11 July 2018

Seoul, Korea 


\section{Introduction}

We consider the minimal singlet seesaw extension of the SM, where the production and decay properties of the sterile neutrino are governed by its mass and mixing with the active neutrinos. We do not want to go into the specific details of neutrino mass models, but keep our discussion generic, regardless of whether the sterile neutrinos are Majorana or pseudo-Dirac particles. In this sense, our results are applicable to all low-scale singlet seesaw models with the SM gauge group, including the minimal type-I seesaw, as well as its variants, such as inverse linear and generalized seesaw. Due to the active-sterile neutrino mixing, a light neutrino flavor eigenstate $\left(v_{\ell}\right)$ is a linear combination of the light $\left(v_{m}\right)$ and heavy $\left(N_{m}\right)$ neutrino mass eigenstates: $v_{\ell} \simeq$ $U_{\ell m} v_{m}+V_{\ell n} N_{n}$ where $U$ is the $3 \times 3$ light neutrino mixing matrix (which is same as the PMNS mixing matrix to leading order, if we ignore the non-unitarity effects), and $V \simeq M_{D} M_{N}^{-1}$ is the active-sterile mixing parameter. The charged-current (CC) interaction in the lepton sector is then given in $[1,2,3,4,11,7,8,9,13,11,12,13]$ Similarly, the relevant Yukawa interaction is given by $\mathscr{L}_{Y} \supset-Y_{D_{\ell m}} \bar{L}_{\ell} \phi N_{m}+$ H.c. where $L$ and $\phi$ are the $S U(2)_{L}$ lepton and Higgs doublets, respectively. After electroweak (EW) symmetry breaking by the vacuum expectation value (VEV) of the Higgs doublet, $\left\langle\phi^{0}\right\rangle=v$, we get the Dirac mass term $M_{D}=v Y_{D}$. So the Yukawa coupling of the sterile neutrino to the SM Higgs is given by $Y_{D}=V M_{N} / v$, which is also suppressed by $V$. For simplicity, we will assume that only the lightest heavy neutrino mass eigenstate (denoted here simply by $N$ ) is kinematically accessible at colliders, and denote the corresponding mixing parameter as simply $V_{\ell N}$, which is the only free parameter in our phenomenological analysis, apart from the sterile neutrino mass $M_{N}$. From the charged current, neutral current and Yukawa interactions, we see that there are three decay modes for the sterile neutrino, if kinematically allowed: $N \rightarrow \ell^{-} W^{+}, v_{\ell} Z$, $v_{\ell} h$, where $h$ is the SM Higgs boson (the only physical scalar remnant of the doublet $\phi$ ). The corresponding partial decay widths are respectively given in [1] The total decay width is just the sum of the above three partial widths for each flavor and summed over all lepton flavors. If $N$ is a Majorana particle, the charge-conjugate modes, namely, $\ell^{+} W^{-}, \bar{v} Z$ and $\bar{v} h$ are also allowed, so there is an additional factor of 2 . For $M_{N}<M_{W}$, none of these two-body decay modes are kinematically allowed. In this case, the sterile neutrino will have three-body decays dominantly mediated by the SM gauge bosons. The corresponding partial decay widths when the off-shell SM gauge bosons decay leptonically and hadronically are given in [1].

\section{Analysis}

The new Yukawa interaction gives rise to a new decay mode for the SM Higgs, $h \rightarrow N v$, if kinematically allowed. Depending on the $N$ decay, we will have different final states. In this section, we will examine the leptonic final states $2 \ell 2 v$, which can arise from either $N \rightarrow \ell_{1}^{-} W^{+(*)} \rightarrow$ $\ell_{1}^{-} \ell_{2}^{+} v$ (with both $\ell_{1}=\ell_{2}$ and $\ell_{1} \neq \ell_{2}$ possibilities) or $N \rightarrow v Z^{(*)} \rightarrow v \ell^{-} \ell^{+}$. The important thing to note here is that these final states mimics the SM process $h \rightarrow W W^{*} \rightarrow 2 \ell 2 v$, and therefore, enhance the $h \rightarrow W W^{*}$ signal strength, while suppress the other SM decay modes, with respect to the SM predictions. It is worth mentioning here that the $h \rightarrow W W^{*}$ channel has the second largest branching fraction (22\%) in the SM for $M_{h}=125 \mathrm{GeV}$ and is a good candidate for studying Higgs boson properties. Before going into the experimental details, we would like to point out that due 

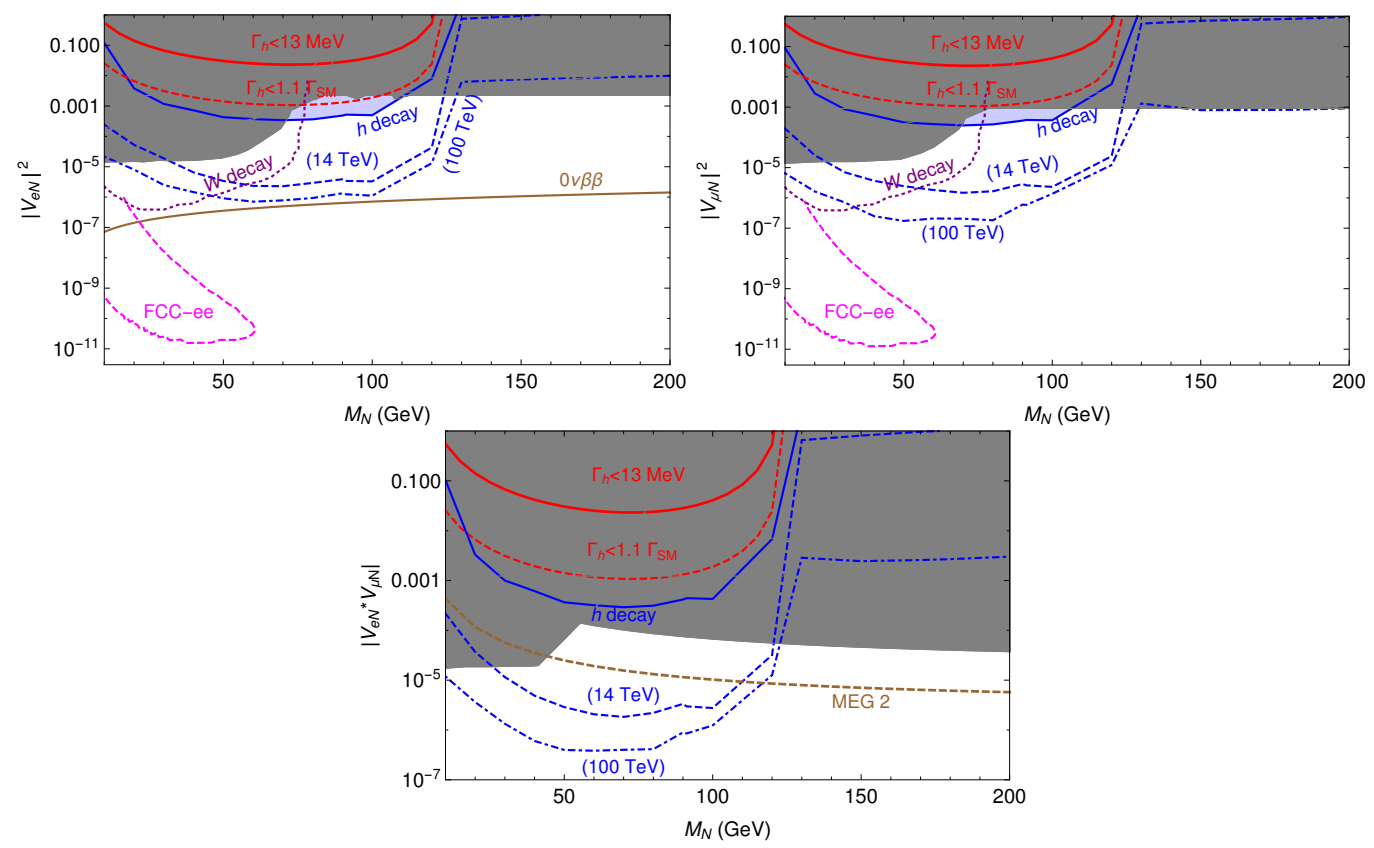

Figure 1: Upper bound on the mixing angle from the $h \rightarrow 2 \ell 2 v$ channel at the LHC. The left panel in the upper row stands for $2 \mu 2 v$, the right panel shows the result for $2 e 2 v$ final state, and the lower row stands for $e \mu 2 v$ channel. The shaded regions in each panel is experimentally excluded from a combination of low and high-energy searches for sterile neutrinos. For comparison, we also show the corresponding current/future limits from a few other relevant experiments. For details, see [1].

to the new Yukawa interaction, the total decay width of the Higgs boson is also enhanced with respect to its SM predicted value [1]. From the LHC studies of Higgs boson off-shell production in gluon fusion and vector boson fusion processes, an upper limit on the total width of the SM Higgs boson of $\Gamma_{h}<13 \mathrm{MeV}$ at 90\% CL has been derived by CMS and ATLAS (see [1] for references). This implies an upper limit on the Yukawa coupling, and hence, on the mixing parameter $\left|V_{\ell N}\right|^{2}$ [1]. With future precision Higgs measurements, this limit could be further improved. For instance, up to $10 \%$ precision in Higgs total width can be achieved at a $100 \mathrm{TeV} p p$ collider: $\Gamma_{h}<1.1 \Gamma_{\mathrm{SM}}$, which corresponds to a limit on the mixing parameter as shown by the red dashed curve [1]. A future lepton collider can achieve an accuracy of up to 5\% (2.5\% with the luminosity upgrade [1]). The limits on the mixings are given in Fig. 1 corresponding explanations on the results, necessary cuts and detailed results, see[1].

\section{Conclusion}

In this analysis we found that the $2 \ell 2 v$ final states from the Higgs decay to $N$ can set bounds on the corresponding mixings. We notice that the $0 v 2 \beta$ bound on the $\left|V_{e N}\right|^{2}$ is the strongest which can be extremely difficult to cover come even at the $100 \mathrm{TeV}$ collider. However, in the $\left|V_{\mu N}\right|^{2}$, the $100 \mathrm{TeV}$ bound is the strongest. From the Higgs decay, roughly $110 \mathrm{GeV}$ RHN can be probed below the electroweak precision limit. The $e \mu 2 v$ final state is strongly constrained by the $\mu \rightarrow e \gamma$ 
process from MEG. However, at the $100 \mathrm{TeV}$ collider, the bound on the mixing angle can be better than the the MEG limit for $M_{N} \leq 120 \mathrm{GeV}$. The other experimental bounds can be observed in [14].

\section{References}

[1] A. Das, P. S. B. Dev and C. S. Kim, "Constraining Sterile Neutrinos from Precision Higgs Data," Phys. Rev. D 95, no. 11, 115013 (2017) doi:10.1103/PhysRevD.95.115013 [arXiv:1704.00880 [hep-ph]].

[2] P. S. Bhupal Dev, R. Franceschini and R. N. Mohapatra, "Bounds on TeV Seesaw Models from LHC Higgs Data,” Phys. Rev. D 86, 093010 (2012) doi:10.1103/PhysRevD.86.093010 [arXiv:1207.2756 [hep-ph]].

[3] C. G. Cely, A. Ibarra, E. Molinaro and S. T. Petcov, "Higgs Decays in the Low Scale Type I See-Saw Model,” Phys. Lett. B 718, 957 (2013) doi:10.1016/j.physletb.2012.11.026 [arXiv:1208.3654 [hep-ph]].

[4] A. M. Gago, P. HernÃąndez, J. Jones-PÃl'rez, M. Losada and A. Moreno Brice Ãśo, "Probing the Type I Seesaw Mechanism with Displaced Vertices at the LHC, "Eur. Phys. J. C 75, no. 10, 470 (2015) doi:10.1140/epjc/s10052-015-3693-1 [arXiv:1505.05880 [hep-ph]].

[5] A. Das, Y. Gao and T. Kamon, "Heavy Neutrino Search via the Higgs boson at the LHC," arXiv:1704.00881 [hep-ph].

[6] A. Das, "Searching for the minimal Seesaw models at the LHC and beyond," Adv. High Energy Phys. 2018, 9785318 (2018) doi:10.1155/2018/9785318 [arXiv:1803.10940 [hep-ph]].

[7] S. Antusch, E. Cazzato and O. Fischer, "Sterile neutrino searches at future $e^{-} e^{+}, p p$, and $e^{-} p$ colliders,” Int. J. Mod. Phys. A 32, no. 14, 1750078 (2017) doi:10.1142/S0217751X17500786 [arXiv:1612.02728 [hep-ph]].

[8] S. Antusch, E. Cazzato and O. Fischer, "Higgs production from sterile neutrinos at future lepton colliders,” JHEP 1604, 189 (2016) doi:10.1007/JHEP04(2016)189 [arXiv:1512.06035 [hep-ph]].

[9] A. Das and N. Okada, "Inverse seesaw neutrino signatures at the LHC and ILC," Phys. Rev. D 88, 113001 (2013) doi:10.1103/PhysRevD.88.113001 [arXiv:1207.3734 [hep-ph]].

[10] A. Das, P. Konar and S. Majhi, "Production of Heavy neutrino in next-to-leading order QCD at the LHC and beyond," JHEP 1606, 019 (2016) doi:10.1007/JHEP06(2016)019 [arXiv:1604.00608 [hep-ph]].

[11] A. Das, "Searching for the minimal Seesaw models at the LHC and beyond," Adv. High Energy Phys. 2018, 9785318 (2018) doi:10.1155/2018/9785318 [arXiv:1803.10940 [hep-ph]].

[12] A. Das, P. S. Bhupal Dev and N. Okada, "Direct bounds on electroweak scale pseudo-Dirac neutrinos from $\sqrt{s}=8$ TeV LHC data,” Phys. Lett. B 735, 364 (2014) doi:10.1016/j.physletb.2014.06.058 [arXiv:1405.0177 [hep-ph]].

[13] A. Das, P. Konar and S. Majhi, "Production of Heavy neutrino in next-to-leading order QCD at the LHC and beyond,” JHEP 1606, 019 (2016) doi:10.1007/JHEP06(2016)019 [arXiv:1604.00608 [hep-ph]].

[14] A. Das and N. Okada, "Bounds on heavy Majorana neutrinos in type-I seesaw and implications for collider searches,” Phys. Lett. B 774, 32 (2017) doi:10.1016/j.physletb.2017.09.042 [arXiv:1702.04668 [hep-ph]]. 